\title{
A Practical System and Procedure Enabling Battery Trading in Real-World Industry
}

\author{
Victor K. Y. Chan* and Sok Lei Leong \\ School of Business, Macao Polytechnic Institute, Rua de Luís Gonzaga Gomes, Macao, China \\ ${ }^{*}$ Corresponding author
}

\begin{abstract}
The article documents a practical system and its procedure to estimate batteries' remaining energy storage capacities, which are determinants of batteries' economic values, in a bid to facilitate fair-price battery trading. Such trading is in strong demand given today's burgeoning electric vehicles of which batteries when discharged can be replaced with charged ones through "trade and swap" much faster than can be recharged.
\end{abstract}

Keywords-battery; battery trading; remaining energy storage capacity; economic value

\section{INTRODUCTION}

Being able to store electric energy, batteries have been applied in industry for centuries [1] to power electrical or electronic instruments or devices, industrial equipment and recently electric vehicles [2]. However, in particular, rechargeable batteries' remaining energy storage capacities and thus their remaining operational lifetimes decline as they are continuously or repeatedly charged and discharged to supply electric energy where a battery's remaining energy storage capacity is the upper limit on the amount of energy storable in the battery upon completion of a charging cycle or the upper limit on the amount of energy storable in and subsequently releasable by the battery upon completion of a charging cycle. As batteries' numbers of charging and recharging cycles undergone and their ages increase, their remaining energy storage capacities decrease [3], and eventually they approach the ends of their usage lives when their remaining energy storage capacities drop to levels precluding them from serving as practical energy storage any longer.

Therefore, in case of any occasion of battery trading, batteries' remaining energy storage capacities are key determinants of the batteries' economic values. However, there have not been any readily implementable systems and procedures proposed to estimate batteries' remaining energy storage capacities and thus evaluate their economic values in order to distinguish "less" worn-out batteries of larger remaining energy storage capacities from "more" worn-out batteries in terms of their price tags. As such, there has not been any practicable battery trading operation in the real world mainly due to the lack of ways for "more" worn-out battery sellers to be paid commensurately less, "less" worn-out sellers to be paid appropriately more, or owners to financially make up for the economic value disparity when exchanging their "more" worn-out batteries for "less" worn-out ones.
Nevertheless, there is a strong market demand for trading discharged batteries for charged batteries in the context of electric vehicles [2] in view of the much shorter time to replace discharged batteries with charged batteries than to charge discharged batteries in electric vehicles in situ. In case of such replacement, electric vehicles can continue with their journeys within short periods of replacement time whilst discharged batteries unloaded from the electric vehicles can subsequently be charged for replacing discharged batteries of the same or other electric vehicles. [4]

At the very best, battery exchange operations have been in place in some regions. In a typical battery exchange operation, all the batteries legally belong to the same business entity, and subscribers to such an operation are entitled to hold a certain quantity of such batteries and swap batteries to exchange discharged ones being held for charged ones available at some battery exchange stations. In other words, no transfer of ownership is conducted during any battery exchange, so no evaluation of batteries' economic values is necessary. [4]

Based on a patent pending of the authors, this article proposes a system and its procedure to estimate batteries' remaining energy storage capacities and thus indicatively evaluate and/or proportionately compare their economic values, realizing battery trading. The philosophy is that batteries' remaining energy storage capacities and their remaining operational lifetimes decrease as, probably inter alia, the batteries' self-discharge, ages, average depths of discharge experienced, and/or numbers of charging and/or discharging cycles undertaken increase [5] where a battery's self-discharge is the percentage decrease in the battery's state of charge under the absence of any external discharge circuit within a fixed period of time upon the completion of a charging cycle, a battery's depth of discharge is the one's complement of the battery's state of charge, i.e. one minus the battery's state of charge, and a battery's state of charge is the proportion of the amount of energy stored in the battery to the battery's remaining energy storage capacity. Alternatively, a battery's self-discharge can be presented as in the formula below:

$$
D=\frac{\sigma_{0}-\sigma_{\mathrm{F}}}{\sigma_{0}} \times 100 \%
$$

where $D=$ the battery's self-discharge, 
$\sigma_{0}=$ the battery's state of charge at the beginning of the fixed period of time, and

$\sigma_{\mathrm{F}}=$ the battery's state of charge at the end of the fixed period of time.

\section{Procedure to Estimate Batteries’ Remaining ENERGY STORAGE CAPACITIES}

\section{A. Derivation of the Mathematical Relationship}

The system is capable of recording batteries' remaining energy storage capacities retrospectively over their usage lives and is capable of measuring and/or recording one or more parameters of batteries as follows:

- the index of a battery's remaining energy storage capacity (in a way similar to that in section B below),

- the battery's self-discharge within a fixed period of time upon the completion of a charging cycle,

- the difference between the timestamp of the battery's first charging and/or discharging cycle and the current time,

- the battery's average depth of discharge experienced, and

- the battery's number of charging and/or discharging cycles undertaken.

Before estimating any battery's energy storage capacity, the system records the known (i.e., historical) remaining energy storage capacities and the corresponding known values of one or more aforementioned parameters for multiple cases of multiple batteries at multiple points in time, and deduces the mathematical relationship between a battery's known remaining energy storage capacity and the known values of its aforementioned parameters. In practice, this deduction of the mathematical relationship is performed and updated only periodically and independently of the estimation of any battery's remaining energy storage capacity.

With the aforesaid mathematical relationship so deduced, the remaining procedure to estimate a particular battery's unknown remaining energy storage capacity, which is indicative of and proportionate to the battery's economic value, proceeds as in the flowchart in Figure I. Sections B and C below contain elaboration on the two major sub-procedures in the flowchart.

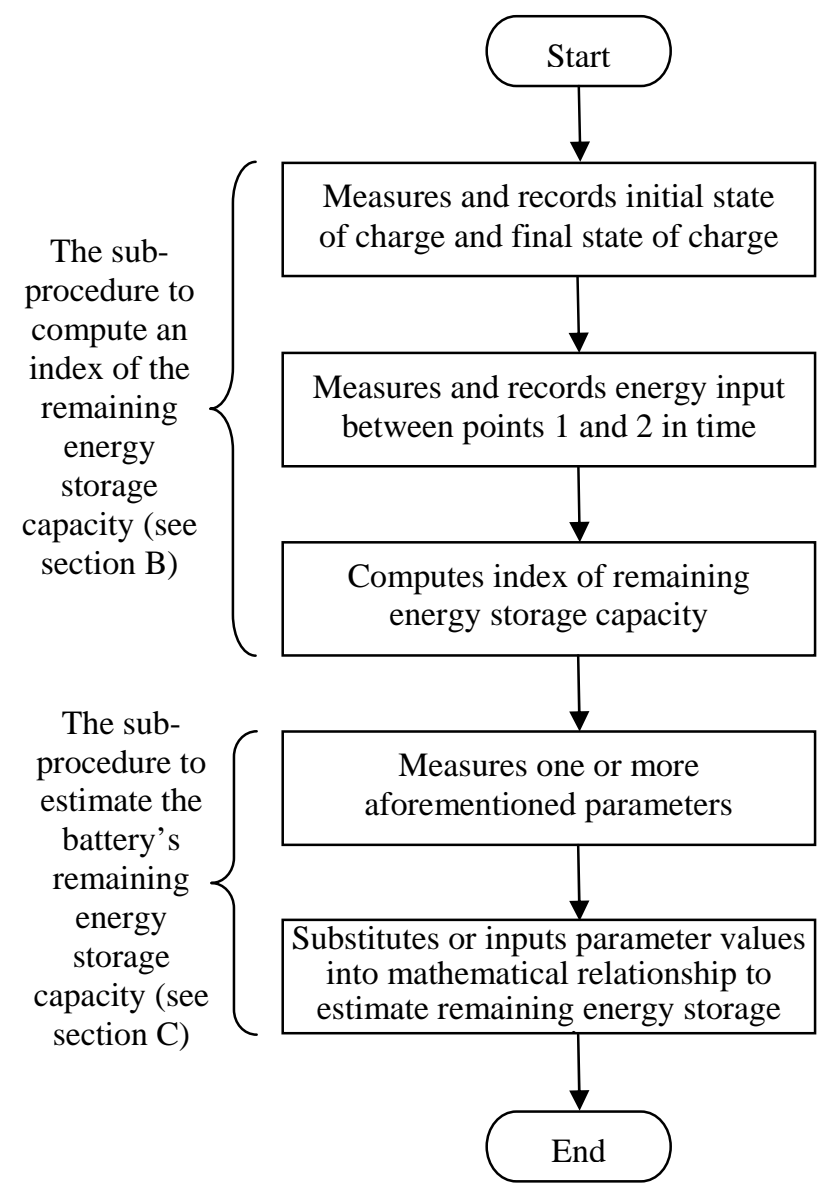

FIGURE I. FLOWCHART: TO ESTIMATE A BATTERY'S UNKNOWN REMAINING ENERGY STORAGE CAPACITY GIVEN THE MATHEMATICAL RELATIONSHIP.

\section{B. Computing an Index of the Battery's Remaining Energy Storage Capacity}

The system measures and/or records the battery's initial state of charge and final state of charge respectively at point 1 and a subsequent point 2 in the battery's charging cycle. At the same time, the amount of energy inputted into the battery between these two points in time is gauged and/or recorded. Consequently, the system computes an index of the battery's remaining energy storage capacity as in (1) below:

$$
C=\frac{E}{S_{\mathrm{F}}-S_{0}}
$$

where $C=$ the index of the battery's remaining energy storage capacity,

$E=$ the energy inputted into the battery between points 1 and 2,

$S_{\mathrm{F}}=$ the battery's final state of charge, and

$S_{0}=$ the battery's initial state of charge. 


\section{Estimating the Battery's Remaining Energy Storage Capacity}

The system substitutes or inputs the measured values of the battery's aforementioned parameters into the deduced mathematical relationship so as to give rise to an estimate of the battery's unknown remaining energy storage capacity.

\section{STRUCTURE OF THE SYSTEM}

As shown in Figure II, the proposed system consists of six modules, namely,

- a State of Charge Recording Module (SCRM), which measures and/or records a battery's initial state of charge and final state of charge respectively at point 1 and a subsequent point 2 in the battery's charging cycle,

- an Energy Input Measurement Module (EIMM), which measures and/or records the amount of energy inputted into the battery between points 1 and 2 in the battery's charging cycle,

- a Remaining Energy Storage Capacity Index Computation Module (RESCICM), which computes according to (1) the index of a battery's remaining energy storage capacity based on the initial state of charge and the final state of charge as outputted by SCRM, and the amount of energy inputted into the battery between points 1 and 2 in the battery's charging cycle as outputted by EIMM,

- a Parameter Recording Module (PRM), which measures and/or records one or more parameters (as enumerated in section II-A above) of a battery and/or the timestamp of the battery's first charging and/or discharging cycle, the index of the battery's remaining energy storage capacity being a parameter outputted by the RESCICM,

- a Mathematical Relationship Derivation Module (MRDM), which deduces the mathematical relationship between a battery's known remaining energy storage capacity and the known values of one or more parameters (as enumerated in section II-A above) of the battery, and

- a Remaining Energy Storage Capacity Computation Module (RESCCM), which computes a battery's remaining energy storage capacity based on what the PRM measures and/or records.

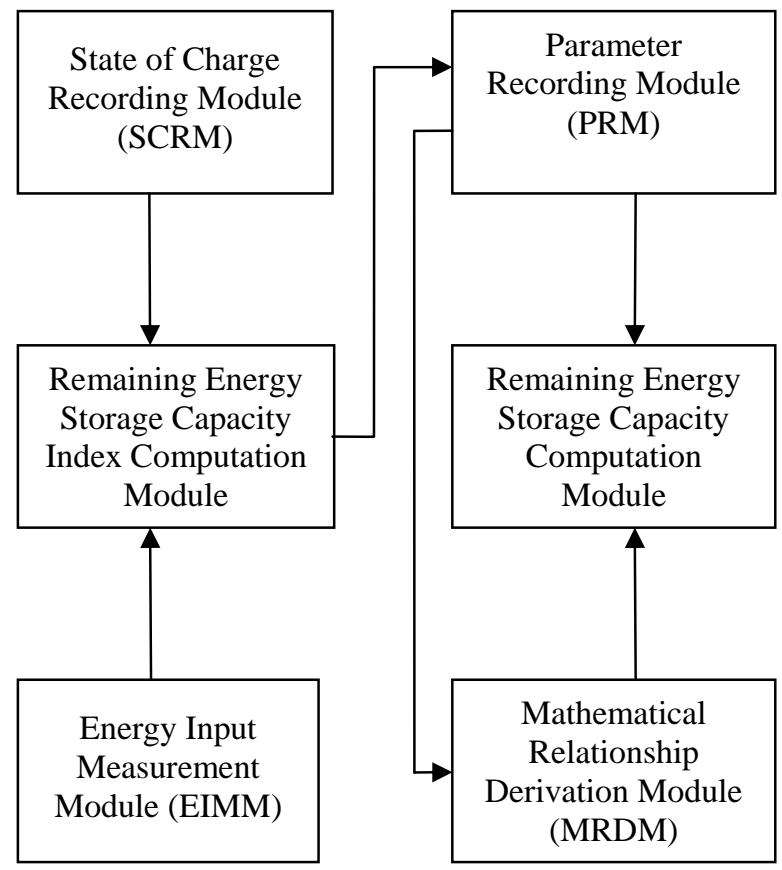

FIGURE II. STRUCTURE OF THE PROPOSED SYSTEM.

The SCRM, EIMM, RESCICM, and PRM are typically but not necessarily attached to the battery concerned, and the MRDM and RESCCM either are so attached or reside on a central computer system. If on a central computer system, the MRDM and RESCCM can typically receive data from the PRMs of a large number of batteries via wireless data communication links or occasionally plugged-in wired communication links.

The MRDM's and RESCCM's respective deduction of the mathematical relationship and estimation of a battery's remaining energy storage capacity can typically be done by means of mathematical regression and neural networks.

If mathematical regression is adopted, a battery's remaining energy storage capacity at a certain point in time serves as the dependent variable $y$, one or more parameters (as enumerated in section II-A above and measured and/or recorded by PRM) of the battery at that point in time as the independent variables $x_{1}, x_{2}, \ldots$, and $x_{m}$. A regression equation as follows is formulated.

$$
\hat{y}_{i}=f\left(x_{1, i}, x_{2, i}, \ldots, x_{m, i} ; \beta_{1}, \beta_{2}, \ldots, \beta_{p}\right)
$$

where $f: \mathbb{R}^{m} \rightarrow \mathbb{R}$ is an assumed, known function that can be expressed in a mathematical expression and is dependent on the type of regression adopted,

$$
\begin{aligned}
& \hat{y}_{i}=\text { an estimate of } y \text { in case } i \text { as given by (2), } \\
& x_{1, i}=\text { the value of } x_{1} \text { in case } i, \\
& x_{2, i}=\text { the value of } x_{2} \text { in case } i,
\end{aligned}
$$


$x_{m, i}=$ the value of $x_{m}$ in case $i$,

$\beta_{1}, \beta_{2}, \ldots$, and $\beta_{p}$ are some unknown, real-number regression coefficients in the mathematical expression of $f$,

$p$ is a natural number, and

$\mathbb{R}$ is the real number set.

In the MRDM, (2) is then optimized by varying the values of $\beta_{1}, \beta_{2}, \ldots$, and $\beta_{p}$ in accordance with the type of regression adopted and based on the known (i.e., historical) remaining energy storage capacities and the corresponding known values of the parameters for multiple cases. Substituting the optimized values of $\beta_{1}, \beta_{2}, \ldots$, and $\beta_{p}$ into (2) gives an optimized (2), which is the mathematical relationship to deduce. Thereafter, for any battery with unknown remaining energy storage capacity $y$ at a certain point in time, an estimate $\hat{y}$ of $y$ can be obtained in RESCCM by substituting into the optimized (2) the battery's values of the parameters $x_{1, i}, x_{2, i}, \ldots$, and $x_{m, i}$ at that point in time as measured and/or recorded by the PRM.

If neural networks are used, a battery's remaining energy storage capacity at a certain point in time is a neural network's output $y$, one or more parameters (as enumerated in section IIA above and measured and/or recorded by PRM) of the battery at that point in time are the inputs $x_{1}, x_{2}, \ldots$, and $x_{m}$. A certain neural network structure (e.g., fully connected backpropagation) and certain types of activation functions (e.g., logistic) in the nodes (or neurons) therein are opted for. Then, in MRDM, supervised learning based on the known (i.e., historical) remaining energy storage capacities and the corresponding known values of the parameters for multiple cases is carried out to optimize the activation functions (or more exactly, optimize some local parameters in the activation functions) in the nodes of the neural network. This ends up with an optimized neural network with optimized activation functions in the nodes, such an optimized neural network being the mathematical relationship to deduce. Thereafter, for any battery with unknown remaining energy storage capacity $y$ at a certain point in time, an estimate $\hat{y}$ of y can be obtained in RESCCM as the output from the optimized neural network when inputting into the optimized neural network the battery's values of the parameters $x_{1}, x_{2}, \ldots$, and $x_{m}$ at that point in time as measured and/or recorded by the PRM.

\section{ACKNOWLEDGMENT}

This article was supported by Grants (004/2013/A and others to be assigned) of the Science and Technology Development Fund, the Government of Macao Special Administrative Region.

\section{REFERENCES}

[1] American Chemical Society, The Columbia Dry Cell Battery: A National Historic Chemical Landmark. Washington, DC: American Chemical Society, 2005.

[2] Y.-W. Wang, "Locating battery exchange stations to serve tourism transport: A note," Transportation Research Part D: Transport and Environment, vol. 13, pp. 193-197, May 2008.
[3] M. Majima, S. Ujiie, E. Yagasaki, K. Koyama, and S. Inazawa, "Development of long life lithium ion battery for power storage," J. Power Sources, vol. 101, pp. 53-59, October 2001.

[4] L.-Y. Tseng, "Combined parking meter and electric-vehicle battery charger with remote status receiver,” US Patent US5563491 A.

[5] N. Omar, M. A. Monem, Y. Firouz, J. Salminen, J. Smekens, O. Hegazy, H. Gaulous, G. Mulder, P. Van den Bossche, T. Coosemans, J. Van Mierlo, "Lithium iron phosphate based battery - Assessment of the aging parameters and development of cycle life model,” Applied Energy, vol. 113, pp. 1575-1585, January 2014. 